\title{
Análise da percepção de um grupo de idosos a respeito de seu handicap auditivo antes e após o uso do aparelho auditivo
}

\author{
Analysis of the perception of a group of seniors about their hearing handicap before and after \\ hearing aid use
}

Ana Cristina Guarinello

Shirlei Biano Marcelos ${ }^{1}$

Angela Ribas ${ }^{1}$

Jair Mendes Marques ${ }^{1}$

\section{Resumo}

Introdução: A presbiacusia, ou perda auditiva causada pelo envelhecimento, pode gerar dificuldades comunicativas, psicológicas e sociais nos indivíduos idosos, com impactos profundos em sua vida social e familiar. Objetivo: Este estudo visou analisar a percepção de um grupo de idosos a respeito de seu handicap auditivo antes e após o uso do aparelho auditivo. Métodos: Foi aplicado um questionário a 29 idosos oriundos de um hospital particular da cidade de Curitiba, PR. Neste estudo, os idosos responderam a perguntas referentes às consequências emocionais causadas pela perda auditiva e sobre os efeitos sociais dessa perda. O questionário foi respondido antes da colocação do aparelho auditivo e, em um segundo momento, após um ou dois anos de uso. Resultados: Verificou-se a existência de diferenças significativas entre os handicaps médios antes e após a protetização $(\mathrm{p}=0,0000)$. Desta forma, houve diminuição significativa do handicap auditivo após uso da prótese auditiva no grupo estudado. Conclusão: Constatouse que os idosos obtiveram bom aproveitamento geral da prótese auditiva após um ano de sua utilização, o que gerou impacto positivo na qualidade de vida e na percepção do seu handicap auditivo antes e depois do uso da prótese.

\section{Abstract}

Introduction: Presbycusis or hearing loss caused by aging can generate communicative psychological and social problems in the elderly, with profound impacts on their social and family life. Objective: This study aimed to analyze the perception of a group of seniors about their hearing handicap before and after using a hearing aid. Methods: A questionnaire was administered to 29 elderly coming from a private hospital in the city of Curitiba, Brazil. In this study the elderly responded to questions regarding the emotional consequences caused by hearing loss and about the social effects of this loss. The questionnaire was completed prior to placing the hearing aid and in a second stage, after one or two years of use. Results: We found significant differences between
Palavras-chave: Auxiliares de Audição. Perda Auditiva. Idoso. Qualidade de Vida. Reabilitação.
Key words: Hearing Aids. Hearing Loss. Elderly. Quality of Life. Rehabilitation.

Programa de Pós-graduação em Distúrbios da Comunicação, Faculdade de Ciências Biológicas e da Saúde. Universidade Tuiuti do Paraná. Curitiba, PR, Brasil. 
the average handicap before and after using the device $(p=0.0000)$. Thus there was significant decrease of hearing handicap after hearing aid use in the study group. Conclusion: It was found that the elderly generally made good use of hearing aids after a year of use, which had a positive impact on the quality of life and perception of their auditory handicap before and after use of the prosthesis.

\section{INTRODUÇÃo}

A perda auditiva relacionada ao envelhecimento é conhecida como presbiacusia. ${ }^{1}$ Esta se caracteriza normalmente por uma perda auditiva bilateral para tons de alta frequência, devido a mudanças degenerativas e fisiológicas no sistema auditivo com o avançar da idade. Ocorre diminuição da capacidade de mitose de certas células, acúmulo de pigmentos intracelulares (lipofucsina) e alterações químicas no fluido intercelular. ${ }^{2}$

As mudanças mais significantes nos limiares auditivos começam a surgir entre os 40 e 50 anos de idade e continuam progredindo até os 80 anos, sendo que mais da metade dos sujeitos que se encontram na faixa etária entre 50 e 60 anos já apresentam algum tipo de comprometimento dos limiares auditivos. ${ }^{3,4}$

A presbiacusia tem como principal consequência um declínio na capacidade comunicativa do indivíduo, o que tende a isolá-lo e privá-lo das fontes de informação e comunicação, maximizando ainda mais as alterações causadas pelo envelhecimento. ${ }^{5}$ Dessa forma, é comum que os idosos com perda auditiva apresentem redução na inteligibilidade de fala. Esse fato, em geral, compromete seriamente seu processo de comunicação verbal e pode fazer com que apresentem sintomas de depressão e demência, ${ }^{6}$ afastando-os do meio social e causando impactos profundos em suas vidas, tendo em vista a implicação direta da perda auditiva com a qualidade de vida dessa população. ${ }^{7-9}$

A perda auditiva nos idosos significa mais do que diminuição da sensibilidade auditiva; é também a impossibilidade de comunicação mais efetiva, ${ }^{10-12}$ que acarreta dificuldades para falar ao telefone na presença de ruídos externos, ouvir a televisão e o rádio, participar de reuniões familiares ou profissionais e conversar com mais de uma pessoa ao mesmo tempo. ${ }^{13,14}$

Estudos ${ }^{15,16}$ demonstram que a implantação de um programa de reabilitação auditiva e o uso de aparelhos auditivos podem minimizar essas reações psicossociais e proporcionar melhora da qualidade de vida dessas pessoas, incluindo a redução da incapacidade auditiva e da desvantagem auditiva (bandicap). Cabe esclarecer que a incapacidade está relacionada à falta de habilidade para a percepção de fala em ambientes ruidosos e o bandicap implica os aspectos não auditivos que impedem que os sujeitos desempenhem adequadamente seu papel na sociedade. ${ }^{17}$

A Organização Mundial da Saúde ${ }^{18}$ define handicap como sendo a desvantagem consequente de uma deficiência ou incapacidade que limitaria ou impediria o indivíduo de desempenhar atividades consideradas normais para a idade, como atividades culturais e sociais. Assim, esse handicap é considerado a percepção do próprio indivíduo a respeito de sua limitação auditiva, o que afeta seu estilo de vida, sua família, sua situação social e seu trabalho.

Apesar de os aparelhos auditivos minimizarem os impactos nos sujeitos que possuem alguma perda, seu uso não é capaz de restaurar a audição normal. Eles se destinam a fornecer a maior quantidade de informações acústicas possíveis, mas não são capazes de modificar o comportamento do usuário. ${ }^{19} \mathrm{O}$ benefício adquirido com uso desses aparelhos pode ser definido como vantagens ou ganhos obtidos pelo usuário com a amplificação sonora ${ }^{20}$ e pode ser medido como positivo, negativo ou neutro, dependendo do efeito que o aparelho exerce sobre o desempenho de cada um. ${ }^{21}$ 
Estudos $^{22}$ demonstram que o uso da prótese auditiva é essencial para manter a saúde física e mental do idoso, pois lhe permite participar melhor da comunidade em que está inserido e do convívio familiar, trazendo uma percepção de seu bandicap auditivo.

Com base nessas considerações, o objetivo do presente estudo foi analisar a percepção de um grupo de idosos a respeito de seu handicap auditivo antes e após o uso do aparelho auditivo.

\section{METODOLOGIA}

Trata-se de uma pesquisa descritiva e comparativa, cujos participantes foram idosos portadores de deficiência auditiva característica de presbiacusia que adquiriram suas próteses auditivas em um hospital privado, localizado na cidade de Curitiba, PR. Utilizou-se para análise desta variável a classificação de Lloyd et al. ${ }^{23}$

Participaram da pesquisa 29 idosos que procuraram o serviço de otorrinolaringologia do hospital e, após a realização de exames audiológicos, como audiometria completa, impedanciometria, BERA e otoemissões acústica transiente e distorção, foram encaminhados para seleção e adaptação de prótese auditiva.

Dos 29 sujeitos idosos que participaram do estudo, 13 eram do sexo masculino e 16 do sexo feminino. Suas idades variavam entre 60 e 94 anos, com idade média de 71,3 anos. Os sujeitos pertenciam às classes socioeconômicas $\mathrm{B}$ e C. O critério de inclusão na pesquisa relacionouse ao tipo de perda incluindo configuração e grau, e os idosos que possuíam diagnóstico de doença neurológica ou psíquica foram excluídos da amostra.

Os dados foram coletados de março a dezembro de 2010. Durante o processo de seleção dos aparelhos, um examinador, estudante de Fonoaudiologia devidamente orientado por um professor doutor da área, aplicou o questionário Hearing Handicap Inventory for Adults (HHIA) ${ }^{24-26}$ a cada idoso, individualmente. O mesmo questionário foi reaplicado pelo mesmo examinador depois da adaptação das próteses, em um prazo que variou de um a dois anos de uso das mesmas. O questionário HHIA é composto por 25 perguntas, das quais 13 exploram as consequências emocionais da deficiência auditiva e 12 se referem aos efeitos sociais e situacionais dessa deficiência.

O handicap auditivo em adultos é avaliado segundo os critérios de pontuação com perguntas que trazem as seguintes alternativas: sim (cada resposta vale 4 pontos), não (sem pontuação) e às vezes (cada resposta vale 2 pontos). Segundo o critério para análise das respostas desse questionário, ${ }^{24}$ quanto maior for a pontuação, maior é a percepção do indivíduo em relação a sua desvantagem auditiva, ou seja, com relação às dificuldades geradas pela deficiência auditiva.

Os dados obtidos neste estudo foram digitados em planilha eletrônica e analisados estatisticamente por meio do teste $t$ Student para dados pareados, ao nível de significância de 0,05. O software utilizado foi o Statistica Release 7, licenciado para o Departamento de Informática da Universidade Tuiuti do Paraná, sob o no 5ANGUUUZU7MGJMU9URKC.

Além da análise dos dados obtidos no questionário apontados na tabela 1 , procedemos à análise qualitativa de algumas questões especialmente selecionadas: 1) a perda auditiva faz você evitar grupos de pessoas?; 2) a perda auditiva deixa você irritado(a)?; 3) a perda auditiva, de alguma forma, limita sua vida pessoal e social?; 4) a perda auditiva faz você sentir-se triste e deprimido? Cabe esclarecer que essas questões foram escolhidas por permitirem compreender a percepção dos indivíduos a respeito de suas limitações auditivas e do quanto essas limitações afetam suas relações pessoais e sociais.

A pesquisa foi aprovada pelo Comitê de Ética Institucional sob o n ${ }^{\circ}$ CEP/UTP-0076/2007, e todos os participantes do estudo assinaram o Termo de Consentimento Livre e Esclarecido, conforme os preceitos éticos estabelecidos para realização de pesquisas envolvendo seres humanos. 


\section{RESULTADOS}

Com relação ao grau da perda auditiva verificou-se que $79 \%$ da amostra estudada possuíam perda auditiva neurossensorial de grau leve a moderado e de configuração descendente, característica de presbiacusia. Em $21 \%$ da amostra, foi detectada perda auditiva mista de grau leve a moderado de configuração descendente. Todos os idosos usavam as próteses auditivas por um ano, em média.

$\mathrm{Na}$ tabela 1 são apresentados os dados coletados com relação à percepção do handicap auditivo, antes e depois do uso da prótese auditiva.

Tabela 1 - Média das respostas da percepção do handicap auditivo antes e depois da protetização (n=29). Curitiba, PR, 2010.

\begin{tabular}{lcccc}
\hline \multicolumn{1}{c}{ Variáveis } & $\mathrm{n}$ & média & $\mathrm{dp}$ & $\mathrm{p}$ \\
\hline Handicap antes do uso do AASI & 29 & $59 \%$ & $23 \%$ & 0,0000 \\
Handicap pós-uso do AASI & 29 & $23 \%$ & $24 \%$ & 0,0000 \\
\hline
\end{tabular}

AASI= aparelho de amplificação sonora individual; $\mathrm{dp}=$ desvio-padrão.

Por meio do teste $t$ Student, para dados pareados ao nível de significância de 0,05 (5\%), foram verificadas diferenças significativas entre os handicaps médios antes e após a protetização ( $\mathrm{p}=0,0000)$. Como é possível visualizar na tabela 1, houve diminuição significativa do bandicap auditivo após uso da prótese auditiva no grupo estudado, o que permite inferir que um bom nível de satisfação foi alcançado pelos usuários.
A tabela 2 apresenta os dados referentes às questões qualitativas analisadas individualmente. Apesar de as respostas dos participantes apontarem para a melhora na sua qualidade de vida após a adaptação do aparelho auditivo, por meio do teste de diferença de proporções ao nível de significância de $0,05(5 \%)$, essa melhora foi significativa apenas para a questão $3(p=0,0217)$.

Tabela 2 - Análise da percepção dos idosos a respeito de suas limitações auditivas ( $\mathrm{n}=29)$. Curitiba, PR, 2010.

\begin{tabular}{lccc}
\hline \multicolumn{1}{c}{ Questões } & \multicolumn{2}{c}{ Sim } & P \\
\cline { 2 - 4 } & Antes & Pós & \\
\hline 1. A perda auditiva faz você evitar grupo de pessoas? & 12 & 6 & 0,0940 \\
2. A perda auditiva deixa você irritado(a)? & $(41,4 \%)$ & $(20,7 \%)$ & \\
3. A perda auditiva, de alguma forma, & 9 & 7 & 0,5588 \\
limita sua vida pessoal e social? & $(31,0 \%)$ & $(24,1 \%)$ & \\
4. A perda auditiva faz você sentir-se triste e deprimido? & 19 & 10 & 0,0217 \\
& $(65,5 \%)$ & $(34,5 \%)$ & \\
& 12 & 6 & 0,0940 \\
\hline
\end{tabular}




\section{DISCUSSÃO}

O tipo e o grau da perda auditiva identificados na amostra estudada são característicos da presbiacusia, o que está de acordo com outros estudos que demonstram que há uma prevalência maior de perda auditiva neurossensorial descendente, de grau leve a moderado, na população idosa. ${ }^{2,6} \mathrm{Nesses}$ casos, o uso da prótese auditiva é recomendado como melhor recurso de reabilitação. ${ }^{12}$

Os dados apresentados sugerem que $\mathrm{O}$ uso do aparelho auditivo, quando realizado adequadamente, tende a melhorar a qualidade de vida de pessoas idosas, pois diminui os efeitos da perda auditiva sobre a comunicação e as relações sociais dessas pessoas. Estes achados estão de acordo com outros autores, para os quais a perda auditiva nos idosos é acompanhada de mudanças psicológicas que impedem uma comunicação eficiente, prejudicando a habilidade de manter relações sociais. ${ }^{27}$

Nos dados apresentados, os idosos referem melhora em sua qualidade de vida, com redução de queixas importantes, como evitar grupos de pessoas, ficar irritado ou sentir-se triste em função da deficiência auditiva. A literatura afirma que, na população portadora de presbiacusia, a maior dificuldade de comunicação é sentida em situações em que várias pessoas estão conversando ao mesmo tempo. ${ }^{2,26}$ De acordo com a tabela 2, 12 pessoas antes de usar as próteses auditivas evitavam reunir-se em grupos por causa da perda auditiva e sentiam-se tristes e deprimidas, e 19 afirmavam que a perda auditiva limitava sua vida social. Depois do uso da prótese, em ambos os casos, houve melhora desses aspectos.

A prótese auditiva, quando bem adaptada, resgata de alguma forma o interesse em participar do âmbito social. ${ }^{28}$ Este estudo demonstrou que o uso das próteses auditivas teve impacto positivo sobre a qualidade de vida dos idosos, pois reduziu a percepção do bandicap auditivo tanto no âmbito emocional quanto no social.
Cabe esclarecer que, apesar de os valores prescritos apontarem para um ganho significativo na maioria dos pesquisados, quando os dados são analisados individualmente, verifica-se que alguns usuários ainda apresentam insatisfação e dificuldades. Isso evidencia que a percepção do handicap auditivo pode variar muito de indivíduo para indivíduo, a depender do gênero, idade, fator socioeconômico, aceitação da deficiência e do próprio prazo de reaplicação do questionário, que foi aplicado em média um ano após o uso da prótese, o que de certo modo pode ter influenciado os resultados desta análise e ser um fator de limitação deste estudo. Outro fator de limitação do estudo é o número reduzido de sujeitos da pesquisa.

Assim, é importante reavaliar mais idosos e com uma periodicidade mais frequente e perceber se a percepção do bandicap auditivo melhora após mais tempo de uso da prótese. Considerase imprescindível, então, o acompanhamento fonoaudiológico, a manutenção do aparelho auditivo, a orientação e o aconselhamento para assegurar o sucesso da reabilitação auditiva, evitando e amenizando as dificuldades que possam eventualmente acontecer no decorrer do tempo.

\section{CONCLUSÃO}

Constatou-se, neste estudo, que os idosos obtiveram bom aproveitamento geral da prótese auditiva após um ano de sua utilização, o que gerou impacto positivo na sua vida social e na percepção do seu handicap auditivo antes e depois do uso da prótese. Além disso, evidenciou-se que o uso da amplificação auditiva diminuiu a percepção desses sujeitos a respeito de suas limitações na vida social e pessoal, tornando-os menos tristes e deprimidos e fazendo com que participassem mais da vida em grupo.

Assim, o uso da prótese auditiva em idosos portadores de perda auditiva característica de presbiacusia torna-se fundamental. Cabe esclarecer essa população a respeito do uso funcional das próteses, de preferência por meio de 
programas de reabilitação audiológica que levem em conta tanto as orientações gerais a respeito do bom uso e funcionamento das próteses, mas também as queixas de cada sujeito com relação à piora de sua qualidade de vida.

\section{REFERÊNCIAS}

1. Braga SR. Próteses Auditivas: conhecimentos essenciais para atender bem o paciente com prótese auditiva. São Paulo: Editora Pulso; 2003. Capítulo 1, Considerações básicas sobre o processo de indicação, seleção e adaptação de próteses auditivas; p. 11-5.

2. Almeida MR, Guarinello AC. Reabilitação audiológica em pacientes idosos. Rev Soc Bras Fonoaudiol 2009;14(2):247-55.

3. Brant LJ, Fozard JL. Age changes in pure-tone hearing thresholds in a longitudinal study of normal human aging. J Acoust Soc Am 1990;88(2):813-20.

4. Soncini F, Costa MJ, Oliveira TMT. Perfil audiológico de indivíduos na faixa etária entre 50 e 60 anos. Fono Atual 2004;28(7):21-9.

5. Ferreira MIDC, Signor RC. O perfil do idoso usuário de prótese auditiva: um estudo da satisfação. Rev Fonoaudiol Bras 2006;4(2):1-2.

6. Marques ACO, Kozlowski L, Marques JM. Reabilitação auditiva no idoso. Rev Bras Otorrinolaringol 2004;70(6):806-11.

7. Rosa MRD, Dante G, Ribas A. Programa de orientação a usuários de prótese auditiva $\mathrm{e}$ questionários de auto-avaliação: importantes instrumentos para uma adaptação auditiva efetiva. Arq Int Otorrinolaringol 2006;10(3):220-7.

8. Seidman MD, Ahmad N, Joshi D, Seidman J, Thawani S, Quirk WS. Age-related hearing loss and its association with reactive oxygen species and mitochondrial DNA damage. Acta Otolaryngol 2004;552:16-24.

9. Lessa AH, Costa MJ, Becker KT, Vaucher AVA. Satisfação de usuários de próteses auditivas, com perda auditiva de graus severo e profundo. Arq Int Otorrinolaringol 2010;14(3):338-45.

10. Callahan EH, Paris B. Midlife periodic health exam in the primary care practice. Geriatrics 1997;52(10):60-79.

11. Teixeira AR, Almeida LG, Jotzs GP, Barba MC. Qualidade de vida de adultos e idosos pós adaptação de próteses auditivas. Rev Soc Bras Fonoaudiol 2008;13(4):357-61.
Conclui-se, portanto, que o trabalho fonoaudiológico nesta faixa etária da população é complexo, e que o fonoaudiólogo deve conhecer o processo de envelhecimento para obter melhores resultados na reabilitação auditiva.

12. Almeida K, Freire KGM. Seleção e adaptação de prótese auditiva para o idoso. In: Almeida K, Iorio MCM. Próteses auditivas. Fundamentos teóricos e aplicações clínicas. São Paulo: Lovise; 2003. p. 385-407.

13. Hétu R, Riverin L, Lalande NM, Getty L, St-Cyr C. The reluctance to acknowledge hearing difficulties among hearing-impaired workers. Br J Audiol 1990;24(4):265-76.

14. Paschoal SMP. Qualidade de Vida na Velhice. In: Freitas EV, Py L, Neri AL, Cançado FAX, Gorzoni ML, Rocha SM, editores. Tratado de Geriatria e Gerontologia. 2. ed. Rio de Janeiro: Guanabara Koogan; 2006. p.79-84.

15. Andrade CF, Blasca WQ. A satisfação do usuário de aparelho de amplificação sonora individual com a tecnologia digital. Salusvita 2005;24(2):257-74.

16. Ruchel CV, Carvalho CR, Guarinello AC. A eficiência de um programa de reabilitação audiológica em idosos com presbiacusia e seus familiares. Rev Soc Bras Fonoaudiol 2007;12(2):92-8.

17. Russo ICP, Almeida K. O Processo de Reabilitação Audiológica do Deficiente Auditivo Idoso. In: Marchesan IQ, Bolaffi C, Gomes ICD, Zorzi JL, organizadores. Tópicos de Fonoaudiologia. São Paulo: Lovise; 1995. p. 430-47.

18. World Health Organization. International classification of impairments, disabilities and handicaps: a manual of classifications relating to consequences of disease. Genebra: WHO; 1980.

19. Almeida K. Seleção e adaptação de próteses auditivas em adultos. In: Ferreira LP, Befi-Lopes DM, Limongi SCO, organizadores. Tratado de Fonoaudiologia. São Paulo: Roca; 2005. p. 669-79.

20. Gil D. Treinamento auditivo formal em adultos com deficiência auditiva. [tese de Doutorado]. São Paulo: Escola Paulista de Medicina na Universidade Federal de São Paulo; 2006.

21. Jerger J. What determines benefit from hearing aid? J am Acad Audiol 2000;11(8):368. 
22. Veiga LR, Merlo ARC, Mengue SS. Satisfação com a prótese auditiva na vida diária em usuários do Sistema de Saúde do Exército. Rev Bras Otorrinolaringol 2005;71(1):67-73.

23. Lloyd LL, Gladstone VS, Kaplan H. Audiometric interpretation: a manual of basic audiometry. Baltimore: University Park Press; 1978.

24. Audiometria tonal, logoaudiometria e medidas de imitância acústica: orientações dos Conselhos de Fonoaudiologia para o laudo audiológico. Brasília: CFFA; 2009. 19 p.

25. Ventry IM, Weinstein BE. The hearing handicap inventory for the elderly: a new tool. Ear Hear 1982;3(3):128-34.
26. Aiello CP, Lima II, Ferrari DV. Validity and reliability of the hearing handicap inventoty for adults. Braz J Otorhinolaryngol 2011;77(4):132-8.

27. Gonçalves CHO, Mota PHM. Saúde auditiva para a terceira idade: comentários sobre um programa de atenção à saúde auditiva. Disturb Comum 2002;13(2):335-49.

28. Russo ICP, Almeida K, Freire GMF. Seleção e adaptação da prótese auditiva para o idoso. In: Almeida K, Iório MCM. Próteses auditivas: fundamentos teóricos e aplicações clínicas. 2. ed. São Paulo: Lovise; 2003. p. 385-410. 